\title{
Respiratory muscle wasting in the ICU: is it time to protect the diaphragm?
}

\author{
Theodoros Vassilakopoulos
}

\begin{abstract}
Mechanical ventilation is a life-saving 'therapeutic' intervention for patients with respiratory failure. The vast majority of critically ill patients are mechanically ventilated (MV) and a significant part of time is spent in weaning patients from mechanical ventilation. It is during weaning that the diaphragm becomes so important becoming a major pathophysiological determinant of weaning failure or success. $^{12}$
\end{abstract}

Various insults can render the diaphragm weak in critically ill patients such as sepsis, multiorgan failure and/or inflammation, electrolyte disturbances, hyperinflation, to name a few out of a long list. ${ }^{3}$ Extensive animal research ${ }^{4}$ and few human studies ${ }^{5-8}$ have provided evidence that controlled mechanical ventilation (CMV), a form of ventilation where the ventilator assumes all the work of breathing and the respiratory muscles are theoretically completely unloaded, can also cause dysfunction of the diaphragm, an entity named ventilator-induced diaphragm dysfunction (VIDD). ${ }^{9}$

VIDD has been described in the laboratory in previously healthy animals subjected to $\mathrm{CMV}^{4}$ and in brain-dead patients free of infection and other derangements so as to be eligible for organ donation. ${ }^{5-8}$ The mechanisms of VIDD are not fully elucidated, but include muscle atrophy (lysosomal, autophagy, cysteine protease and ubiquitin-proteasome activation having all been described), oxidative stress, structural injury (disrupted myofibrils, lipid vacuoles and abnormally small and disrupted mitochondria), myofibre remodelling and mitochondrial dysfunction. The animal data have shown that these insults result in the decline of the pressuregenerating capacity of the diaphragm, that is, reduced diaphragmatic contractility. The extent to which this happens in humans and thus is clinically relevant is largely unknown.

Much of the uncertainty in proving the concept that mechanical ventilation per se

Correspondence to Professor Theodoros Vassilakopoulos, Pulmonary Unit, Critical Care Department, Medical School, National \& Kapodistrian University of Athens, Evangelismos Hospital, 45-47 Ipsilandou str, Athens 10675, Greece; tvassil@med. uoa.gr

can cause contractile dysfunction of the diaphragm derives from the difficulty in accurately studying the performance of the diaphragm in critically ill patients. Diaphragm contractility in MV patients can be assessed using various techniques which are all demanding.

The simplest approach is to indirectly assess diaphragmatic function by measuring the change in endotracheal tube pressure induced by application of bilateral magnetic twitch stimulation of the phrenic nerves during airway occlusion (TwPtr). Jaber and colleagues used this approach in MV intensive care unit (ICU) patients and compared them with patients undergoing endoscopy. ${ }^{6}$ TwPtr decreased progressively over time relative to its initial baseline value in the MV group, with a statistically significant reduction after 3-4 days of $\mathrm{MV}$ reaching a 32\% reduction by the end of the evaluation period (5-6 days of MV). ${ }^{6}$

Magnetic phrenic nerve stimulation can be used with direct measurement of the transdiaphragmatic pressure development with the use of oesophageal and gastric balloons connected to pressure transducers. Using this method, various groups of investigators demonstrated that the diaphragmatic contractility is reduced in MV ICU patients. ${ }^{10-13}$ However, this technique cannot distinguish between impaired phrenic nerve function, abnormal neuromuscular transmission and intrinsic abnormalities in the diaphragm muscle itself. Consequently, using this technique, it is impossible to figure out whether intrinsic contractile weakness of diaphragm muscle fibres occurs in critically ill MV patients. Furthermore, this technique has never been used in braindead organ donors wherein the 'isolated' effects of CMV can to some extent be studied (as free as possible from the various insults to the diaphragm from the ICU environment).

A more accurate yet invasive approach to study diaphragmatic contractile performance in the critically ill is to use isolated permeabilised single diaphragm fibres from biopsies during surgery. However, because diaphragm biopsies are obtained from the belly of the muscle, the fibre ends are disrupted, which precludes electrical activation of the fibres.
Therefore, the fibres have to be permeabilised and subsequently to be activated with exogenous calcium. Using this technique, Hooijman and colleagues provided us with the only proof of concept (until now) study for contractility decline due to VIDD in brain-dead organ donor patients on mechanical ventilation. ${ }^{14}$ These investigators compared the contractile performance (maximum isometric force, cross-bridge kinetics and $\mathrm{Ca}^{2+}$ sensitivity) of permeabilised single muscle fibres derived from diaphragms of brain-dead organ donors undergoing an average of $26 \pm 5 \mathrm{~h}$ of CMV (range 6-48) with those derived from control subjects exposed to $2 \mathrm{~h}$ of $\mathrm{MV}$ while undergoing lung cancer surgery. They failed to detect any differences between the two groups and concluded that $26 \mathrm{~h}$ of CMV in humans does not affect contractile performance of sarcomeres. ${ }^{14}$ Thus, until now, we had no proof that mechanical ventilation can cause contractile dysfunction of the diaphragm despite the suggestive animal data.

Hussain and colleagues provide us with this important missing proof-of-concept information on the effects of VIDD on diaphragmatic contractility in humans and explore underlying mechanisms. ${ }^{15}$ These investigators obtained diaphragm biopsies from 13 subjects undergoing cardiac surgery (control group) and 12 brain-dead organ donors (CMV group). They used the isolated myofibril preparation, which allows for more accurate measurements of protein contractile activity by avoiding the kinds of artefactual effects of noncontractile intracellular elements that occur in single fibre preparations. Brain-dead organ donor patients had been MV 47.6 $\pm 5.0 \mathrm{~h}$ (range 12-74), respectively, in contrast to cardiac surgery patients who were exposed to short periods of mechanical ventilation (for 2$4 \mathrm{~h})$. Specific force generation of diaphragm myofibrils was measured with atomic force cantilevers. Rates of force development, force redevelopment after a shortening protocol and relaxation in fully activated myofibrils (with calcium) were calculated to assess myosin crossbridge kinetics. The brain-dead organ donors on prolonged CMV exhibited reduced maximal active-specific force generation and passive-specific force generation in myofibrils and reduced force redevelopment during activation and in response to imposed shortening. The impairment in contractility was associated with reduced protein levels of myosin heavy chain (slow), troponin-C, troponin-I, troponin-T, tropomyosin and titin. In contrast, no reduction in $\alpha$-actin, 
$\alpha$-actinin or nebulin levels was observed. Thus, we have the first evidence that CMV in humans decreases active diaphragm myofibrillar force generation and passive diaphragm myofibrillar force generation and that this is mediated, in part, by impaired myosin cross-bridge kinetics and decreased myofibrillar protein levels.

Thus, VIDD is one of the (many) causes that can render the diaphragm weak in the ICU environment.

How common is the problem and how can we prevent or even reverse it?

Goligher and colleagues recently described the evolution of diaphragm thickness over time during mechanical ventilation. ${ }^{16}$ They enrolled 107 patients shortly after initiating mechanical ventilation. Diaphragm thickness was measured daily by ultrasound and diaphragm contractile activity was indirectly quantified by the inspiratory thickening fraction of the diaphragm. Over the first week of ventilation, diaphragm thickness decreased by more than $10 \%$ in $47(44 \%)$ and remained unchanged in $47(44 \%) .{ }^{16}$ Low diaphragm contractile activity was associated with rapid decreases in diaphragm thickness. Contractile activity decreased with increasing ventilator driving pressure and controlled ventilator modes. The most likely explanation for the decrease in diaphragm thickness is the development of diaphragmatic atrophy. VIDD may be playing a central role in this process, since low contractile activity of the diaphragm was associated with high pressures delivered by the ventilator and with the use of controlled ventilator modes, which predispose to VIDD. The impressive and clinically important finding was the magnitude of the problem. Within a week, nearly half (44\%) of MV patients developed decreases in diaphragm thickness. This is in accordance with the results of Supinski and Callahan who reported reduced diaphragmatic contractility in the vast majority of the 57 ICU patients they studied.

Thus, in the ICU, the interplay of excessive ventilator support with the other ICU insults causes atrophy of the diaphragm, which makes the diaphragm weak in a time-dependent manner ${ }^{6} 1214$ and thus weaning difficult in a significant proportion of ICU patients.
A rational approach to the problem would be to limit to the extent possible the use of controlled modes of mechanical ventilation and the amount of assist-pressure provided by the ventilator in ICU patients. Of course, this suggestion requires scientific proof with appropriate clinical studies testing this approach both preventively and more importantly therapeutically, that is, after atrophy has developed, to reverse it. The degree of diaphragm loading we should aim in MV patients to prevent or reverse VIDD is not known and has not even be adequately addressed even in animal studies. The results of Goligher et al suggest that excessive loading might also not be beneficial for the diaphragm. Twelve per cent of the patients developed increases in diaphragm thickness associated with high contractile activity of the diaphragm and these patients rather developed weakness of the diaphragm as assessed by the maximum thickening fraction of the diaphragm during a maximal inspiratory effort, which rather excludes hypertrophy as an explanation.

ICU physicians are caring how to protect the lung. Yet, they often forget that one of the two vital pumps, the ventilatory pump, exhibits plasticity and vulnerability and the results of Hussain et al show that the major component of the ventilatory pump, the diaphragm can dysfunction very quickly in the ICU during mechanical ventilation. We need to learn how to protect the diaphragm with large-scale studies similar to those studies that taught us how to protect the lung.

Competing interests None declared.

Provenance and peer review Commissioned; externally peer reviewed.

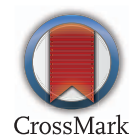

To cite Vassilakopoulos T. Thorax 2016;71:397-398.

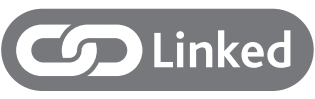

- http://dx.doi.org/10.1136/thoraxjnl-2015-207559

Thorax 2016;71:397-398.

doi:10.1136/thoraxjnl-2016-208354

\section{REFERENCES}

1 Vassilakopoulos T, Zakynthinos S, Roussos C. The tension-time index and the frequency/tidal volume ratio are the major pathophysiologic determinants of weaning failure and success. Am J Respir Crit Care Med 1998;158:378-85.

2 Purro A, Appendini L, De Gaetano A, et al. Physiologic determinants of ventilator dependence in long-term mechanically ventilated patients. Am J Respir Crit Care Med 2000;161:1115-23.

3 Vassilakopoulos T, Zakynthinos S, Roussos C. Respiratory muscles and weaning failure. Eur Respir J 1996;9:2383-400.

4 Vassilakopoulos T. Ventilator-induced diaphragm dysfunction: the clinical relevance of animal models. Intensive Care Med 2008:34:7-16.

5 Hussain SN, Mofarrahi M, Sigala I, et al. Mechanical ventilation-induced diaphragm disuse in humans triggers autophagy. Am J Respir Crit Care Med 2010;182:1377-86.

6 Jaber S, Petrof BJ, Jung B, et al. Rapidly progressive diaphragmatic weakness and injury during mechanical ventilation in humans. Am J Respir Crit Care Med 2011;183:364-71.

7 Levine S, Nguyen T, Taylor N, et al. Rapid disuse atrophy of diaphragm fibers in mechanically ventilated humans. $N$ Engl I Med 2008;358:1327-35.

8 Picard M, Jung B, Liang F, et al. Mitochondrial dysfunction and lipid accumulation in the human diaphragm during mechanical ventilation. Am J Respir Crit Care Med 2012;186:1140-9.

9 Vassilakopoulos T, Petrof BJ. Ventilator-induced diaphragmatic dysfunction. Am I Respir Crit Care Med 2004;169:336-41.

10 Watson AC, Hughes PD, Louise HM, et al. Measurement of twitch transdiaphragmatic, esophageal, and endotracheal tube pressure with bilateral anterolateral magnetic phrenic nerve stimulation in patients in the intensive care unit. Crit Care Med 2001;29:1325-31.

11 Laghi F, Cattapan SE, Jubran A, et al. Is weaning failure caused by low-frequency fatigue of the diaphragm? Am J Respir Crit Care Med 2003;167:120-7.

12 Hermans G, Agten A, Testelmans D, et al. Increased duration of mechanical ventilation is associated with decreased diaphragmatic force: a prospective observational study. Crit Care 2010;14:R127.

13 Supinski GS, Callahan LA. Diaphragm weakness in mechanically ventilated critically ill patients. Crit Care 2013;17:R120.

14 Hooijman PE, Paul MA, Stienen GJ, et al. Unaffected contractility of diaphragm muscle fibers in humans on mechanical ventilation. Am J Physiol Lung Cell Mol Physiol 2014;307:L460-70.

15 Hussain SNA, Cornachione AS, Guichon C, et al. Prolonged controlled mechanical ventilation in humans triggers myofibrillar contractile dysfunction and myofilament protein loss in the diaphragm. Thorax 2016;71:436-45.

16 Goligher EC, Fan E, Herridge MS, et al. Evolution of diaphragm thickness during mechanical ventilation. Impact of inspiratory effort. Am I Respir Crit Care Med 2015;192:1080-8. 